\title{
Flip Distance to some Plane Configurations
}

\author{
Ahmad Biniaz ${ }^{1}$ \\ Cheriton School of Computer Science, University of Waterloo \\ Waterloo, Canada \\ ahmad.biniaz@gmail.com
}

Anil Maheshwari ${ }^{2}$

School of Computer Science, Carleton University

Ottawa, Canada

anil@scs.carleton.ca

\author{
Michiel Smid ${ }^{3}$ \\ School of Computer Science, Carleton University \\ Ottawa, Canada \\ michiel@scs.carleton.ca
}

\begin{abstract}
We study an old geometric optimization problem in the plane. Given a perfect matching $M$ on a set of $n$ points in the plane, we can transform it to a non-crossing perfect matching by a finite sequence of flip operations. The flip operation removes two crossing edges from $M$ and adds two non-crossing edges. Let $f(M)$ and $F(M)$ denote the minimum and maximum lengths of a flip sequence on $M$, respectively. It has been proved by Bonnet and Miltzow (2016) that $f(M)=O\left(n^{2}\right)$ and by van Leeuwen and Schoone (1980) that $F(M)=O\left(n^{3}\right)$. We prove that $f(M)=O(n \Delta)$ where $\Delta$ is the spread of the point set, which is defined as the ratio between the longest and the shortest pairwise distances. This improves the previous bound for point sets with sublinear spread. For a matching $M$ on $n$ points in convex position we prove that $f(M)=n / 2-1$ and $F(M)=\left(\begin{array}{c}n / 2 \\ 2\end{array}\right)$; these bounds are tight.

Any bound on $F(\cdot)$ carries over to the bichromatic setting, while this is not necessarily true for $f(\cdot)$. Let $M^{\prime}$ be a bichromatic matching. The best known upper bound for $f\left(M^{\prime}\right)$ is the same as for $F\left(M^{\prime}\right)$, which is essentially $O\left(n^{3}\right)$. We prove that $f\left(M^{\prime}\right) \leqslant n-2$ for points in convex position, and $f\left(M^{\prime}\right)=O\left(n^{2}\right)$ for semi-collinear points.

The flip operation can also be defined on spanning trees. For a spanning tree $T$ on a convex point set we show that $f(T)=O(n \log n)$.
\end{abstract}

2012 ACM Subject Classification Theory of computation $\rightarrow$ Computational geometry, Mathematics of computing $\rightarrow$ Discrete mathematics

Keywords and phrases flip distance, non-crossing edges, perfect matchings, spanning trees

Digital Object Identifier 10.4230/LIPIcs.SWAT.2018.11

\section{Introduction}

A geometric graph is a graph whose vertices are points in the plane, and whose edges are straight-line segments connecting the points. All graphs that we consider in this paper are geometric. A graph is plane if no pair of its edges cross each other. Let $n \geqslant 2$ be an even integer, and let $P$ be a set of $n$ points in the plane that is in general position (no three points

1 Supported by NSERC and Fields Institute.

2 Supported by NSERC.

3 Supported by NSERC.

cc) (i) Ahmad Binaiz, Anil Maheshwari, and Michiel Smid

16th Scandinavian Symposium and Workshops on Algorithm Theory (SWAT 2018).

Editor: David Eppstein; Article No. 11; pp. 11:1-11:14

Leibniz International Proceedings in Informatics

LIPICS Schloss Dagstuhl - Leibniz-Zentrum für Informatik, Dagstuhl Publishing, Germany 
(a)
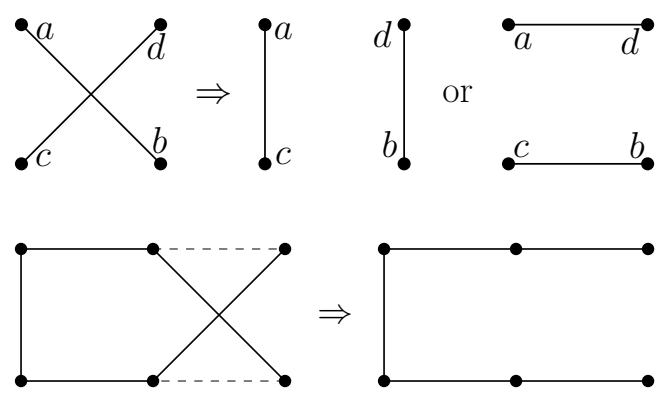

(c)

(b)
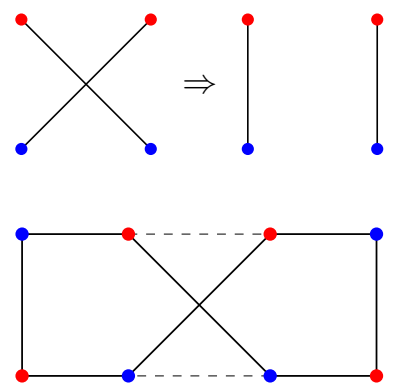

(d)

Figure 1 (a) Two ways to flip a crossing in a monochromatic matching. (b) The only way to flip a crossing in a bichromatic matching. (c) One way to flip a crossing in a monochromatic tree. (d) No way to flip a crossing in a bichromatic Hamiltonian cycle.

on a line). For two points $a$ and $b$ in the plane, we denote by $a b$ the segment with endpoints $a$ and $b$. Let $M$ be a perfect matching on $P$. If two edges in $M$ cross each other, we can remove this crossing by a flip operation. The flip operation (or flip for short) removes two crossing edges and adds two non-crossing edges to obtain a new perfect matching. In other words, if two segments $a b$ and $c d$ cross, then a flip removes $a b$ and $c d$ from the matching, and adds either $a c$ and $b d$, or $a d$ and $b c$ to the matching; see Figure 1(a). Every flip decreases the total length of the edges of $M$, and thus, after a finite sequence of flips, $M$ can be transformed to a plane perfect matching. This process of transforming a crossing matching to a plane matching is referred to as uncrossing or untangling a matching. Motivated by this old folklore result, we investigate the minimum and the maximum lengths of a sequence of flips to reach a plane matching.

To uncross a perfect matching $M$, we say that the sequence $\left(M=M_{0}, M_{1}, \ldots, M_{k}\right)$ is a valid flip sequence if $M_{i+1}$ is obtained from $M_{i}$ by a single flip, and $M_{k}$ is plane. The number $k$ denotes the length of this flip sequence. We define $f(M)$ to be the minimum length of any valid flip sequence to uncross $M$, that is, the minimum number of flips required to transform $M$ to a plane perfect matching. We define $F(M)$ to be the maximum length of any valid flip sequence. As for $F(M)$, one can imagine that an adversary imposes which of the two flips to apply on which of the crossings.

In the bichromatic setting, we are given $n / 2$ red and $n / 2$ blue points and a bichromatic matching, that is a perfect matching in which the two endpoints of every segment have distinct colors. Contrary to the monochromatic setting, there is only one way to flip two crossing bichromatic edges; see Figure 1(b). In the bichromatic setting the adversary can only impose the crossing to flip. Thus, any upper bound on $F(M)$ for monochromatic matchings carries over to bichromatic matchings; this statement is not necessarily true for $f(M)$.

The flip operation can be defined for a spanning tree (resp. a Hamiltonian cycle) analogously, that is, we remove a pair of crossing edges and add two other edges so that the graph remains a spanning tree (resp. a Hamiltonian cycle) after this operation. We define $f(\cdot)$ and $F(\cdot)$ for spanning trees and Hamiltonian cycles, analogously. As shown in Figure 1(c), there is only one way to flip a crossing in a spanning tree (resp. a Hamiltonian cycle). Contrary to the bichromatic matching, it is not always possible to flip a crossing in a bichromatic spanning tree nor in a bichromatic Hamiltonian cycle; see Figure 1(d). 


\subsection{Related Work}

The most relevant works are by van Leeuwen and Schoone [21], and Oda and Watanabe [17] for Hamiltonian cycles, and by Bonnet and Miltzow [7] for matchings. They proved, with elegant arguments, the following results.

- Theorem 1 (van Leeuwen and Schoone, 1981 [21]). For every Hamiltonian cycle $H$ on $n$ points in the plane we have that $F(H)=O\left(n^{3}\right)$.

- Theorem 2 (Oda and Watanabe, 2007 [17]). For every Hamiltonian cycle $H$ on $n$ points in the plane in convex position we have that $f(H) \leqslant 2 n-7$.

As for a lower bound, they presented a Hamiltonian cycle $H$ on $n \geqslant 7$ points in the plane in convex position for which $f(H) \geqslant n-2$.

- Theorem 3 (Bonnet and Miltzow, 2016 [7]). For every perfect matching $M$ on a set of $n$ points in the plane in general position we have that $f(M)=O\left(n^{2}\right)$.

The $O\left(n^{3}\right)$ upper bound of Theorem 1 carries over to perfect matchings. As for lower bounds, Bonnet and Miltzow [7] presented two matchings $M_{1}$ and $M_{2}$ such that $f\left(M_{1}\right)=\Omega(n)$ and $F\left(M_{2}\right)=\Omega\left(n^{2}\right)$. The bound $F(M)=O\left(n^{3}\right)$ holds even if $M$ is a bichromatic matching, while the proof of $f(M)=O\left(n^{2}\right)$ does not generalize for the bichromatic setting.

An alternate definition of an edge flip in a graph is the operation of removing one edge and inserting a different edge such that the resulting graph remains in the same graph class. The edge flip operation has been studied for many different graph classes, in particular, for two given graphs with an equal number of vertices and edges, the number of edge flips required to transform one into another. See the survey by Bose and Hurtado [8] on edge flips in planar graphs both in the combinatorial and the geometric settings, and see $[3,9,14,16]$ for edge flips in triangulations.

A related problem is the compatible matching problem in which we are given two perfect matchings on the same point set and the goal is to transform one to another by a sequence of compatible matchings (two perfect matchings, on the same point set, are said to be compatible if they are edge disjoint and their union is non-crossing). See $[1,2,4,15]$ for recent work on compatible matchings, and [18] for its extension to compatible trees.

\subsection{Our Contribution}

In this paper we decrease the gap between lower and upper bounds for $f(\cdot)$ and $F(\cdot)$ for some input configurations. In Section 2 we show that for every perfect matching $M$, on a set $P$ of $n$ points in the plane, we have $f(M)=O(n \Delta)$ where $\Delta$ is the spread of $P$.

Assume that $P$ is in convex position. In Section 3 we show that for every perfect matching $M$ on $P$ we have that $f(M) \leqslant n / 2-1$ and $F(M) \leqslant\left(\begin{array}{c}n / 2 \\ 2\end{array}\right)$. These bounds are tight as Bonnet and Miltzow [7] showed the existence of two perfect matchings $M_{1}$ and $M_{2}$ on $n$ points in convex position such that $f\left(M_{1}\right) \geqslant n / 2-1$ and $F\left(M_{2}\right) \geqslant\left(\begin{array}{c}n / 2 \\ 2\end{array}\right)$. We also prove that for every spanning tree $T$ on $P$ we have that $f(T)=O(n \log n)$.

In Section 4 we study bichromatic matchings on special point sets. Assume that the points of $P$ are colored red and blue. We prove that, if $P$ is in convex position, then for every perfect bichromatic matching $M$ on $P$ we have that $f(M) \leqslant n-2$. Also, we prove that, if $P$ is semi-collinear, i.e., the blue points are on a straight line, then for every perfect bichromatic matching $M$ on $P$ we have that $f(M)=O\left(n^{2}\right)$. Table 1 summarizes the results. 
Table 1 Upper bounds on the minimum and maximum number of flips ( $\Delta$ is the spread).

\begin{tabular}{|c|c|c|c|c|}
\hline minimum \# of flips & $f(\cdot)$-ge & ral position & \multicolumn{2}{|c|}{$f(\cdot)$-convex position } \\
\hline matchings & $\begin{array}{l}O\left(n^{2}\right) \\
O(n \Delta)\end{array}$ & $\begin{array}{c}{[7]} \\
\text { Theorem } 4\end{array}$ & $n / 2-1$ & Theorem 11 \\
\hline bichromatic matchings & $O\left(n^{3}\right)$ & {$[21]$} & $n-2$ & Theorem 15 \\
\hline trees & $O\left(n^{3}\right)$ & {$[21]$} & $O(n \log n)$ & Theorem 14 \\
\hline Hamiltonian cycles & $O\left(n^{3}\right)$ & {$[21]$} & $2 n-7$ & {$[17]$} \\
\hline \multicolumn{4}{|c|}{ bichromatic matching on semi-collinear points $f(\cdot)=O\left(n^{2}\right)$} & Theorem 17 \\
\hline maximum \# of flips & \multicolumn{2}{|c|}{$F(\cdot)$-general position } & \multicolumn{2}{|c|}{$F(\cdot)$-convex position } \\
\hline matchings/trees/cycles & $O\left(n^{3}\right)$ & {$[21]$} & $\left(\begin{array}{c}n / 2 \\
2\end{array}\right)$ & Theorem 11 \\
\hline
\end{tabular}

\subsection{Preliminaries}

Let $a$ and $b$ be two points in the plane. We denote by $a b$ the straight line-segment between $a$ and $b$, and by $\overline{a b}$ the line through $a$ and $b$. Let $P$ be a set of points in the plane in convex position. For two points $p$ and $q$ in $P$ we define the depth of the segment $p q$ as the minimum number of points of $P \backslash\{p, q\}$ on either side of $\overline{p q}$. A boundary edge is a segment of depth zero, i.e., an edge of the convex hull of $P$. An edge $e$ in a graph $G$ is said to be free if $e$ is not crossed by other edges of $G$.

\section{Minimum Number of Flips}

The spread $\Delta$ of a set of points (also called the distance ratio [11]) is the ratio between the largest and the smallest interpoint distances. It is well known that the spread of a set of $n$ points in the plane is $\Omega(\sqrt{n})$ (see e.g., [19]). In this section, we prove an upper bound on the minimum length of a flip sequence in terms of $n$ and $\Delta$. In fact we prove the following theorem.

- Theorem 4. For every perfect matching $M$ on a set of $n$ points in the plane in general position we have that $f(M)=O(n \Delta)$, where $\Delta$ is the spread of the point set.

For point sets with spread $o(n)$, the upper bound of Theorem 4 is better than the $O\left(n^{2}\right)$ upper bound of Theorem 3. For example, for dense point sets, which have spread $O(\sqrt{n})$, Theorem 4 gives an upper bound of $O(n \sqrt{n})$ on the number of flips. According to [13], dense point sets commonly appear in nature, and they have applications in computer graphics. Valtr and others $[13,19,20]$ have established several combinatorial bounds for dense point sets that improve corresponding bounds for arbitrary point sets.

Let $P$ be a set of $n$ points in the plane with spread $\Delta$. Let $M$ be a perfect matching on $P$. We prove that $M$ can be untangled by $O(n \Delta)$ flips, i.e., $f(M)=O(n \Delta)$. The main idea of our proof is as follows. Let $\mu$ be the minimum distance between any pair of points in $P$. Let $|p q|$ denote the Euclidean distance between two points $p, q \in P$. Since $P$ has spread $\Delta$, we have $|p q| \leqslant \mu \Delta$. For the matching $M$ we define its weight, $w(M)$, to be the total length of its edges. Since $M$ has $n / 2$ edges,

$$
w(M)=\sum_{p q \in M}|p q|=O(n \mu \Delta) .
$$

Recall that a pair of crossing segments can be flipped in two different ways as depicted in Figure 1(a). In the remainder of this section we show that one of these two flip operations 


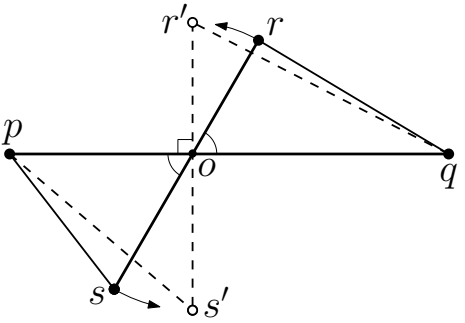

(a)

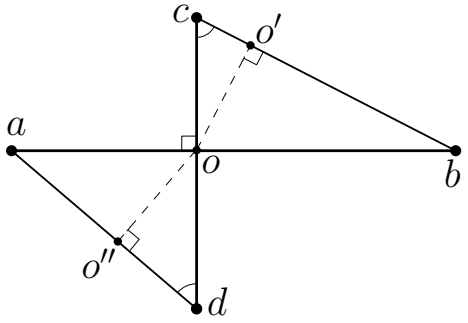

(b)

Figure 2 Illustrations of the proofs of (a) Lemma 7 and (b) Lemma 6 .

reduces $w(M)$ by at least $t \mu$, for some constant $t>0$. Combining this with Equality (1) implies the existence of a flip sequence of length $O(n \Delta)$ that uncrosses $M$.

Take any two crossing edges $p q$ and $r s$ in $M$, and let $o$ be their intersection point. We flip $p q$ and $r s$ to $p s$ and $r q$, if $\angle r o q \leqslant \pi / 2$, and to $p r$ and $q s$, otherwise. In other words, we flip $p q$ and $r s$ to the two edges that face the two smaller angles at $o$. In Lemma 7 we prove that this flip reduces the length of edges by at least $t \mu^{\prime}$, for some constant $t>0$, where $\mu^{\prime}$ is the minimum distance between any pair of points in $\{p, q, r, s\}$. Since the minimum distance between pairs in $\{p, q, r, s\}$ is at least the minimum distance between pairs in $P$, our result follows. We use the following two lemmas in the proof of Lemma 7; we prove these two lemmas later.

- Lemma 5. Let $a b$ and $c d$ be two crossing segments, and let o be their intersection point. Let $\mu^{\prime \prime}$ be the minimum distance between any pair of points in $\{a, b, c, d\}$. If $\angle c o b \leqslant \pi / 3$, then

$$
(|a b|+|c d|)-(|a d|+|c b|) \geqslant \mu^{\prime \prime} .
$$

- Lemma 6. Let $a b$ and $c d$ be two perpendicular segments that cross each other. Let $\mu^{\prime \prime}$ be the minimum distance between any pair of points in $\{a, b, c, d\}$. Then for any constant $t^{\prime} \leqslant(2-\sqrt{2}) / 2$ it holds that

$$
(|a b|+|c d|)-(|a d|+|c b|) \geqslant t^{\prime} \mu^{\prime \prime} .
$$

- Lemma 7. Let $p q$ and rs be two crossing segments, and let o be their intersection point. Let $\mu^{\prime}$ be the minimum distance between any pair of points in $\{p, q, r, s\}$. If $\angle$ roq $\leqslant \pi / 2$, then for some constant $t$ it holds that

$$
(|p q|+|r s|)-(|p s|+|r q|) \geqslant t \mu^{\prime} .
$$

Proof. If $\angle r o q<\pi / 3$, then our claim follows, with $t=1$, from Lemma 5 where $p, q, r, s$ play the roles of $a, b, c, d$, respectively. Assume that $\angle r o q \geqslant \pi / 3$. Observe that $\angle r o q=\angle p o s$.

After a suitable rotation and/or a horizontal reflection and/or relabeling assume that $|p q| \geqslant|r s|, p q$ is horizontal, $p$ is to the left of $q$, and $r$ lies above $p q$. Rotate $r s$ counterclockwise about $o$, while keeping $o$ on this segment, until $r s$ is vertical. See Figure 2(a). After this rotation, let $r^{\prime}$ and $s^{\prime}$ denote the two points that correspond to $r$ and $s$, respectively.

- Claim 8. $\left|r^{\prime} p\right|>|r p| / 2$ and $\left|q s^{\prime}\right|>|q s| / 2$.

We prove only the first inequality of this claim; the proof of the second inequality is analogous. Since $r^{\prime} p$ is the hypotenuse of the right triangle $\triangle r^{\prime} o p$, we have $\left|r^{\prime} o\right| \leqslant\left|r^{\prime} p\right|$. 
Since $\triangle r^{\prime}$ or is isosceles and $\angle r^{\prime}$ or $\leqslant \pi / 6$, we have $\left|r r^{\prime}\right|<\left|r^{\prime} o\right|$, and thus, $\left|r r^{\prime}\right|<\left|r^{\prime} p\right|$. By the triangle inequality we have $|r p| \leqslant\left|r r^{\prime}\right|+\left|r^{\prime} p\right|<2\left|r^{\prime} p\right|$, which implies $\left|r^{\prime} p\right|>|r p| / 2$. This proves Claim 8.

Observe that $\left|r^{\prime} q\right| \geqslant|r q|,\left|p s^{\prime}\right| \geqslant|p s|,\left|r^{\prime} s^{\prime}\right|=|r s|$, and by Claim 8, $\left|r^{\prime} p\right| \geqslant|r p| / 2$ and $\left|q s^{\prime}\right| \geqslant|q s| / 2$. Thus, the minimum distance $\mu^{\prime \prime}$ between any pair of points in $\left\{p, q, r^{\prime}, s^{\prime}\right\}$ is not smaller than half the minimum distance between any pair of points in $\{p, q, r, s\}$, i.e., $\mu^{\prime \prime} \geqslant \mu^{\prime} / 2$. Lemma 6 implies that $\left(|p q|+\left|r^{\prime} s^{\prime}\right|\right)-\left(\left|p s^{\prime}\right|+\left|r^{\prime} q\right|\right) \geqslant t^{\prime} \mu^{\prime \prime}$, for some constant $t^{\prime}>0$, where $p, q, r^{\prime}, s^{\prime}$ play the roles of $a, b, c, d$, respectively. We will see in the proof of Lemma 6 that this inequality is valid for any positive constant $t^{\prime} \leqslant(2-\sqrt{2}) / 2$. Combining these inequalities, we get

$$
\begin{aligned}
(|p q|+|r s|)-(|p s|+|r q|) & \geqslant\left(|p q|+\left|r^{\prime} s^{\prime}\right|\right)-\left(\left|p s^{\prime}\right|+\left|r^{\prime} q\right|\right) \\
& \geqslant \frac{2-\sqrt{2}}{2} \mu^{\prime \prime} \geqslant \frac{2-\sqrt{2}}{4} \mu^{\prime} .
\end{aligned}
$$

Therefore, the claimed inequality in the statement of this lemma is valid for any positive constant $t \leqslant(2-\sqrt{2}) / 4$.

- Note 9. The constants $t^{\prime}=(2-\sqrt{2}) / 2$ and $t=(2-\sqrt{2}) / 4$ in the proofs of Lemmas 6 and 7 are not optimized. To keep our proofs short and simple, we avoid optimizing these constants.

- Note 10. The angle constraint in the statement of Lemma 7 cannot be dropped; the figure to the right shows two crossing segments pq and rs for which $(|p q|+|r s|)-(|p s|+|r q|)$ tends to zero as $\angle$ roq tends to $\pi$.

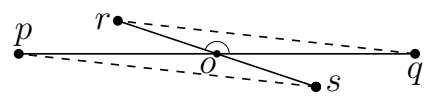

Proof of Lemma 5. We recall the simple fact that the largest side of every triangle always faces the largest angle of the triangle. Since $\angle c o b \leqslant \pi / 3$, we have that $\angle c b o \geqslant \pi / 3$ or $\angle b c o \geqslant \pi / 3$. Without loss of generality assume that $\angle b c o \geqslant \pi / 3$, and thus, $\angle b c o \geqslant \angle c o b$. This implies that $|o b| \geqslant|c b|$. By a similar reasoning, we get that $|o a| \geqslant|a d|$ or $|o d| \geqslant|a d|$. If $|o a| \geqslant|a d|$, then

$$
|a b|+|c d|-(|a d|+|c b|)=(|o a|+|o b|)+|c d|-(|a d|+|c b|) \geqslant|c d| \geqslant \mu^{\prime \prime},
$$

and if $|o d| \geqslant|a d|$, then

$$
|a b|+|c d|-(|a d|+|c b|)=(|o a|+|o b|)+(|o c|+|o d|)-(|a d|+|c b|) \geqslant|o a|+|o c| \geqslant|a c| \geqslant \mu^{\prime \prime}
$$

Proof of Lemma 6. Refer to Figure 2(b) for an illustration of the proof. Let $o$ be the intersection point of $a b$ and $c d$. Let $o^{\prime}$ be the intersection point between $c b$ and the line that is perpendicular to $c b$. Without loss of generality assume that $o b$ is longer than $o c$, i.e., $|o b| \geqslant|o c|$. Then $\angle o c b \geqslant \angle o b c$, and thus, $\angle o c b \geqslant \pi / 4$. Since $\angle o o^{\prime} c=\pi / 2$ and $\angle o c o^{\prime}=\angle o c b \geqslant \pi / 4$, we get that $\angle c o o^{\prime}$ is the smallest angle in the triangle $\triangle o c o^{\prime}$, and thus, $o^{\prime} c$ is its smallest side. By doing some simple algebra we get that $\left|o^{\prime} c\right| \leqslant|o c| / \sqrt{2}$.

Let $o^{\prime \prime}$ be the intersection point between $a d$ and the line that is perpendicular to $a d$. We consider two cases depending on which of $o a$ and $o d$ is longer. 
- $|o a|>|o d|$ : By a similar reasoning as for $o b$ and $o c$ we get that $\left|o^{\prime \prime} d\right| \leqslant|o d| / \sqrt{2}$. Observe that $|o b|>\left|o^{\prime} b\right|$ and $|o a|>\left|o^{\prime \prime} a\right|$. By combining these inequalities we get

$$
\begin{aligned}
(|a b|+|c d|)-(|a d|+|c b|) & =(|o a|+|o b|)+(|o c|+|o d|)-\left(\left|o^{\prime \prime} a\right|+\left|o^{\prime \prime} d\right|\right)-\left(\left|o^{\prime} c\right|+\left|o^{\prime} b\right|\right) \\
& >|o c|+|o d|-\left|o^{\prime \prime} d\right|-\left|o^{\prime} c\right| \geqslant|o c|+|o d|-\frac{|o d|}{\sqrt{2}}-\frac{|o c|}{\sqrt{2}} \\
& =\left(1-\frac{1}{\sqrt{2}}\right)(|o c|+|o d|)=\frac{2-\sqrt{2}}{2}|c d| \geqslant \frac{2-\sqrt{2}}{2} \mu^{\prime \prime} .
\end{aligned}
$$

- $|o a| \leqslant|o d|:$ Again, by a similar reasoning as for $o b$ and $o c$ we get that $\left|o^{\prime \prime} a\right| \leqslant|o a| / \sqrt{2}$. Also, by a similar reasoning as in the previous case we get

$$
\begin{aligned}
(|a b|+|c d|)-(|a d|+|c b|) & \geqslant|o c|+|o a|-\frac{|o a|}{\sqrt{2}}-\frac{|o c|}{\sqrt{2}} \\
& =\frac{2-\sqrt{2}}{2}|c a| \geqslant \frac{2-\sqrt{2}}{2} \mu^{\prime \prime} .
\end{aligned}
$$

Therefore, the claimed inequality in the statement of this lemma is valid for any positive constant $t^{\prime} \leqslant(2-\sqrt{2}) / 2$.

\section{Points in Convex Position}

In this section we study the problem of uncrossing perfect matchings and spanning trees on points in convex position. For perfect matchings, Bonnet and Miltzow [7] exhibited two perfect matchings $M_{1}$ and $M_{2}$ on $n$ points in the plane in convex position such that $f\left(M_{1}\right) \geqslant n / 2-1$ and $F\left(M_{2}\right) \geqslant\left(\begin{array}{c}n / 2 \\ 2\end{array}\right)$. The following theorem provides matching upper bounds for $f(\cdot)$ and $F(\cdot)$.

- Theorem 11. For every perfect matching $M$ on a set of $n$ points in the plane in convex position we have $f(M) \leqslant \frac{n}{2}-1$ and $F(M) \leqslant\left(\begin{array}{c}n / 2 \\ 2\end{array}\right)$.

Proof. The matching $M$ contains $n / 2$ edges. First we prove that $F(M) \leqslant\left(\begin{array}{c}n / 2 \\ 2\end{array}\right)$. Notice that the number of crossings between the edges of $M$ is at most $\left(\begin{array}{c}n / 2 \\ 2\end{array}\right)$. We show that any flip reduces this number by at least one, and thus, our claim follows. Take any pair $a b$ and $c d$ of crossing edges of $M$. Flip this crossing, and let $a c$ and $b d$ be the new edges, after a suitable relabeling. After this flip operation, the crossing between $a b$ and $c d$ disappears. Moreover, any edge of $M$ that crosses $a c$ (or $b d$ ) used to cross $a b$ or $c d$, and any edge of $M$ that crosses both $a c$ and $b d$ used to cross both $a b$ and $c d$. Therefore, the total number of crossings reduces by at least one, and thus, our claim follows.

Now, we prove, by induction on $n$, that $f(M) \leqslant n / 2-1$. If $n=2$, then $M$ has only one edge, and thus, $f(M)=0$. Assume that $n \geqslant 4$. First, we show how to transform $M$, by at most one flip, to a perfect matching $M^{\prime}$ containing a boundary edge, i.e., an edge of the boundary of the convex hull. Let $p_{1}, \ldots, p_{n}$ be the points in clockwise order. Let $p_{i} p_{j}$ be an edge of $M$ with minimum depth $m$. If $m=0$, then $M^{\prime}=M$ is a matching in which $p_{i} p_{j}$ is a boundary edge. Suppose that $m \geqslant 1$. Without loss of generality assume that $i=1$ and $j=m+2$. Let $p_{k}$ be the point that is matched to $p_{2}$ by $M$. Because of the minimality of $m$, the edge $p_{2} p_{k}$ crosses $p_{1} p_{m+2}$. By flipping $p_{2} p_{k}$ and $p_{1} p_{m+2}$ to $p_{1} p_{2}$ and $p_{m+2} p_{k}$ we obtain $M^{\prime}$ in which $p_{1} p_{2}$ is a boundary edge. Let $M^{\prime \prime}$ be the matching on $n-2$ points obtaining from $M^{\prime}$ by removing a boundary edge. By the induction hypothesis, it holds that

$$
f(M) \leqslant 1+f\left(M^{\prime \prime}\right) \leqslant 1+\left(\frac{n-2}{2}-1\right)=\frac{n}{2}-1 .
$$




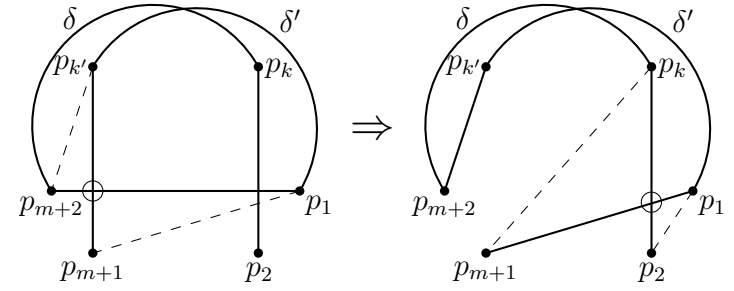

(a)

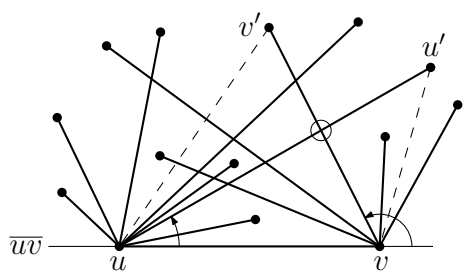

(b)

Figure 3 (a) Illustration of the proof of Lemma 12: Flipping $p_{1} p_{m+2}$ and $p_{m+1} p_{k^{\prime}}$ to $p_{1} p_{m+1}$ and $p_{m+2} p_{k^{\prime}}$, and then flipping $p_{1} p_{m+1}$ and $p_{2} p_{k}$ to $p_{1} p_{2}$ and $p_{k} p_{m+1}$. (b) Illustration of the proof of Lemma 13: $v v^{\prime}$ is the first counterclockwise edge incident on $v$ that is crossed by some edges incident on $u$, and $u u^{\prime}$ is the first counterclockwise edge incident on $u$ that crosses $v v^{\prime}$.

In the rest of this section we study spanning trees. The argument of [21] for Hamiltonian cycles also extends to spanning trees, that is, if $T_{1}$ is a spanning tree on $n$ points in the plane, then $F\left(T_{1}\right)=O\left(n^{3}\right)$. Also, by an argument similar to the one in the proof of Theorem 11 , it can easily be shown that for every spanning tree $T$ on $n$ points in the plane in convex position we have that $F(T)=O\left(n^{2}\right)$. In this section we prove that $f(T)=O(n \log n)$. Recall that a boundary edge is an edge of the boundary of the convex hull.

- Lemma 12. Any spanning tree on a point set in convex position can be transformed, by at most two flips, into a spanning tree containing a boundary edge.

Proof. Let $T$ be a spanning tree on $n$ points in the plane in convex position, and let $p_{1}, \ldots, p_{n}$ be the points in clockwise order. Let $p_{i} p_{j}$ be an edge of $T$ with minimum depth $m$ (recall the definition of depth from Section 1.3). If $m=0$, then $p_{i} p_{j}$ is a boundary edge. Suppose that $m \geqslant 1$. Without loss of generality assume that $i=1$ and $j=m+2$. Because of the minimality of $m$, all edges of $T$ that are incident on $p_{2}, \ldots, p_{m+1}$ cross $p_{1} p_{m+2}$. We consider two cases with $m=1$ and $m>1$.

- $m=1$. In this case $p_{m+1}=p_{2}$ and $p_{m+2}=p_{3}$. Let $\delta$ be the path between $p_{2}$ to $p_{3}$ in $T$, and let $p_{k}$ be the vertex that is adjacent to $p_{2}$ in $\delta$. If $\delta$ contains $p_{1}$, then we flip $p_{1} p_{3}$ and $p_{2} p_{k}$ to $p_{1} p_{2}$ and $p_{3} p_{k}$; this gives a spanning tree in which $p_{1} p_{2}$ is a boundary edge. If $\delta$ does not contain $p_{1}$, then we flip $p_{1} p_{3}$ and $p_{2} p_{k}$ to $p_{2} p_{3}$ and $p_{1} p_{k}$; this gives a spanning tree in which $p_{2} p_{3}$ is a boundary edge.

- $m>1$. Let $\delta$ be the path between $p_{2}$ to $p_{m+2}$ in $T$, and let $p_{k}$ be the vertex that is adjacent to $p_{2}$ in $\delta$. If $\delta$ contains $p_{1}$, then we flip $p_{1} p_{m+2}$ and $p_{2} p_{k}$ to $p_{1} p_{2}$ and $p_{m+2} p_{k}$; this gives the a spanning tree in which $p_{1} p_{2}$ is a boundary edge. Assume that $\delta$ does not contain $p_{1}$. Let $\delta^{\prime}$ be the path between $p_{m+1}$ to $p_{1}$ in $T$, and let $p_{k^{\prime}}$ be the vertex that is adjacent to $p_{m+1}$ in $\delta^{\prime}$; it may be that $k^{\prime}=k$. If $\delta^{\prime}$ contains $p_{m+2}$, then we flip $p_{1} p_{m+2}$ and $p_{m+1} p_{k^{\prime}}$ to $p_{m+1} p_{m+2}$ and $p_{1} p_{k^{\prime}}$; this gives a spanning tree in which $p_{m+1} p_{m+2}$ is a boundary edge. Assume that $\delta^{\prime}$ does not contain $p_{m+2}$. See Figure 3(a). In this case we have that $k^{\prime} \neq k$, because otherwise $T$ would have a cycle. First we flip $p_{1} p_{m+2}$ and $p_{m+1} p_{k^{\prime}}$ to $p_{1} p_{m+1}$ and $p_{m+2} p_{k^{\prime}}$, then we flip $p_{1} p_{m+1}$ and $p_{2} p_{k}$ to $p_{1} p_{2}$ and $p_{k} p_{m+1}$; this gives a spanning tree in which $p_{1} p_{2}$ is a boundary edge.

For the following lemma we do not need the vertices to be in convex position.

- Lemma 13. Let $T$ be a spanning tree containing an edge uv such that every other edge is incident on either $u$ or $v$. Then $f(T) \leqslant \min (\operatorname{deg}(u), \operatorname{deg}(v))-1$, and this bound is tight. 
Proof. After a suitable rotation and/or a horizontal reflection and/or relabeling assume that $\overline{u v}$ is horizontal, $u$ is to the left of $v$, and that $\operatorname{deg}(v) \leqslant \operatorname{deg}(u)$. The edges that are incident on points above $\overline{u v}$ do not cross the edges incident on points below $\overline{u v}$. Thus, the crossings above $\overline{u v}$ can be handled independently of the ones below $\overline{u v}$. Because of symmetry, we describe how to handle the crossings above $\overline{u v}$. See Figure 3(b). We show how to increase, by one flip, the number of free edges that are incident on $v$. By repeating this process, our claim follows. To that end, let $v^{\prime}$ be the first vertex, in counterclockwise order, that is adjacent to $v$, and such that $v v^{\prime}$ is crossed by at least one edge incident on $u$. Let $u^{\prime}$ be the first vertex, in counterclockwise order, that is adjacent to $u$, and such that $u u^{\prime}$ crosses $v v^{\prime}$; see Figure 3(b). Flip this crossing to obtain new edges $v u^{\prime}$ and $u v^{\prime}$. The edge $v u^{\prime}$ is free, because otherwise $u u^{\prime}$ cannot be the first counterclockwise edge that crosses $v v^{\prime}$. Moreover, any edge that is crossed by $u v^{\prime}$ used to be crossed by $u u^{\prime}$. Thus, the number of free edges that are incident on $v$ increases by at least one. By repeating this process, after at most $\operatorname{deg}(v)-1$ iterations, all incident edges on $v$ become free (notice that the edge $u v$ is already free); this transforms $T$ to a plane spanning tree. This proves the first statement of the lemma.

Recall that the statement of this lemma is not restricted to points in convex position, and thus, the vertices of our tight example do not need to be in convex position. To verify the tightness of the bound, consider a tree in which every edge incident on $v$ (except $u v$ ) is crossed by exactly one of the edges incident on $u$, and every edge incident on $u$ crosses at most one of the edges incident on $v$. This tree needs exactly $\operatorname{deg}(v)-1$ flips to be transformed to a plane tree.

- Theorem 14. For every spanning tree $T$ on $n$ points in the plane in convex position we have that $f(T)=O(n \log n)$.

Proof. We present a recursive algorithm that uncrosses $T$ by $O(n \log n)$ flips. As for the base case, if $n \leqslant 3$, then $T$ is plane, and thus, no flip is needed. Assume that $n \geqslant 4$. By Lemma 12 , by at most two flips, we can transform $T$ to a tree $T^{\prime}$ containing a boundary edge $u v$. Contract the edge $u v$ and denote the resulting tree with $n-1$ vertices by $T^{\prime \prime}$; this can be done by removing the vertex $u$ together with its incident edges, and then connecting its neighbors, by straight-line edges, to $v$. We call every such new edge a u-edge. Recursively uncross $T^{\prime \prime}$ with $f\left(T^{\prime \prime}\right)$ flips. During the uncrossing process of $T^{\prime \prime}$, whenever we flip/remove a $u$-edge, we call the new edge that gets connected to $v$ a $u$-edge. After uncrossing $T^{\prime \prime}$ we return the vertex $u$ back and connect it to $v$. Then we remove every $u$-edge $v v^{\prime}$, which is incident on $v$, and connect $v^{\prime}$ to $u$. In the resulting tree, every crossing is between an edge that is incident on $u$ and an edge that is incident on $v$. Thus, after at most $2+f\left(T^{\prime \prime}\right)$ flips, $T$ can be transformed into a tree in which any two crossing edges are incident on $u$ and $v$. Then by Lemma 13 , we can obtain a plane tree by performing at most $\min (\operatorname{deg}(u), \operatorname{deg}(v))-1$ more flips. Notice that the flip operation does not change the degree of vertices, and thus, every vertex in the resulting tree has the same degree as in $T$. Therefore, we have that

$$
\begin{aligned}
f(T) & \leqslant 2+f\left(T^{\prime \prime}\right)+\min (\operatorname{deg}(u), \operatorname{deg}(v))-1 \\
& =1+\min (\operatorname{deg}(u), \operatorname{deg}(v))+f\left(T^{\prime \prime}\right) .
\end{aligned}
$$

It remains to show that $f(T)=O(n \log n)$. To that end, we interpret the above recursion by a union-find data structure with the linked-list representation and the weighted-union heuristic [12, Chapter 21]. The number of flips in the above recursion can be interpreted as the total time for union operations as follows: each time that we contract an edge $u v$ and recurse on a smaller tree we perform at most $1+\min (\operatorname{deg}(u), \operatorname{deg}(v))$ flips. Consider every vertex $x$ of $T$ as a set with $\operatorname{deg}(x)$ elements. Also, assume that all the elements of these sets are pairwise distinct. Thus, we have $n$ disjoint sets of total size $2(n-1)$; this is coming from the fact that $T$ has $n-1$ edges and its total vertex degree is $2(n-1)$. The 


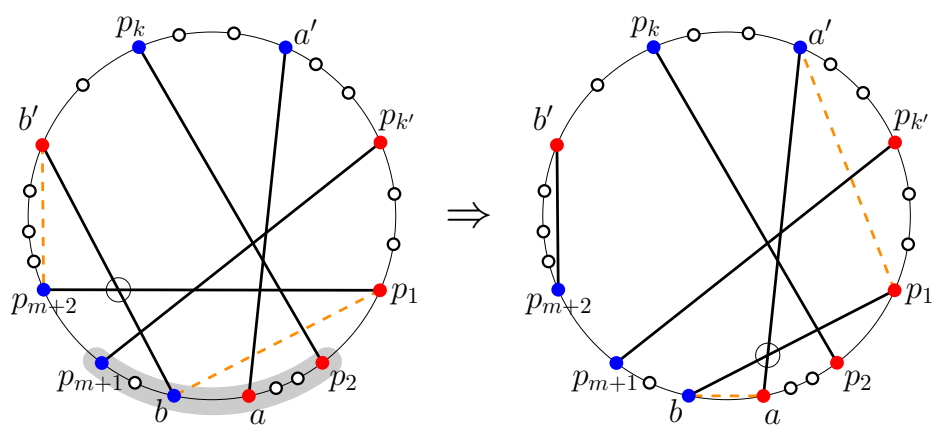

Figure 4 Illustration of the proof of Theorem 15. Flipping $b b^{\prime}$ and $p_{1} p_{m+2}$ to $b^{\prime} p_{m+2}$ and $b p^{\prime}$, and then flipping $b p_{1}$ and $a a^{\prime}$ to $p_{1} a^{\prime}$ and $a b$.

contraction of an edge $u v$ can be interpreted as a union operation of the sets $u$ and $v$ whose cost (number of flips) is at most $1+\min (|u|,|v|)$, where $|x|$ denotes the size of the set $x$. From the union-find data structure we have that the cost of a sequence of $s$ operations on $m$ elements is $O(s+m \log m)$. In our case, the number $m$ of elements is $2(n-1)$, and the number $s$ of union operations (edge contractions) is $n-3$ (no contraction is needed when we hit the base case). Thus, it follows that the total cost (the total number of flips) is $O(n \log n)$.

\section{Bichromatic Matchings}

In this section we study the problem of uncrossing perfect bichromatic matchings for points in convex position and for semi-collinear points. Let $n \geqslant 2$ be an even integer, and let $P$ be a set of $n$ points in the plane, $n / 2$ of which are colored red and $n / 2$ are colored blue. If $P$ is in general position, then for any bichromatic matching $M$ on $P$, the best known upper bound for both $f(M)$ and $F(M)$ is the $O\left(n^{3}\right)$ bound that has been proved in [7,21]. If $P$ is in convex position, the $n / 2-1$ and $\left(\begin{array}{c}n / 2 \\ 2\end{array}\right)$ lower bounds that are shown in [7] for $f(\cdot)$ and $F(\cdot)$, respectively, in the monochromatic setting, also hold in the bichromatic setting. Theorem 11 implies that the $\left(\begin{array}{c}n / 2 \\ 2\end{array}\right)$ bound for $F(\cdot)$ is tight. The following theorem gives an upper bound on $f(\cdot)$ for points in convex position.

Theorem 15. For every perfect bichromatic matching $M$ on $n$ points in the plane in convex position we have that $f(M) \leqslant n-2$.

Proof. Our proof is by induction on $n$. If $n=2$, then $f(M)=0$. Assume that $n \geqslant 4$. First we show how to transform $M$, by at most two flips, to a perfect bichromatic matching $M^{\prime}$ containing a boundary edge. Let $p_{1}, \ldots, p_{n}$ be the points in clockwise order. Let $p_{i} p_{j}$ be an edge of $M$ with minimum depth $m$. If $m=0$, then $M^{\prime}=M$ is a matching in which $p_{i} p_{j}$ is a boundary edge. Suppose that $m \geqslant 1$. Without loss of generality assume that $i=1$, $j=m+2, p_{1}$ is red, and $p_{m+2}$ is blue as in Figure 4. Let $p_{k}$ and $p_{k^{\prime}}$ be the points that are matched to $p_{2}$ and $p_{m+1}$, respectively; it may be that $m+1=2$ and $k^{\prime}=k$. Because of the minimality of $m$, all edges that are incident on points $p_{2}, \ldots, p_{m+1} \operatorname{cross} p_{1} p_{m+2}$. If $p_{2}$ is blue, then by flipping $p_{1} p_{m+2}$ and $p_{2} p_{k}$ to $p_{1} p_{2}$ and $p_{m+2} p_{k}$ we obtain $M^{\prime}$ in which $p_{1} p_{2}$ is a boundary edge. Assume that $p_{2}$ is red. If $p_{m+1}$ is red, then by flipping $p_{1} p_{m+2}$ and $p_{m+1} p_{k^{\prime}}$ to $p_{m+1} p_{m+2}$ and $p_{1} p_{k^{\prime}}$ we obtain $M^{\prime}$ in which $p_{m+1} p_{m+2}$ is a boundary edge. Assume that $p_{m+1}$ is blue. See Figure 4 . To this end, $p_{2}$ and $p_{m+1}$ have different colors, and thus, $m+1 \neq 2$ and $k^{\prime} \neq k$. 


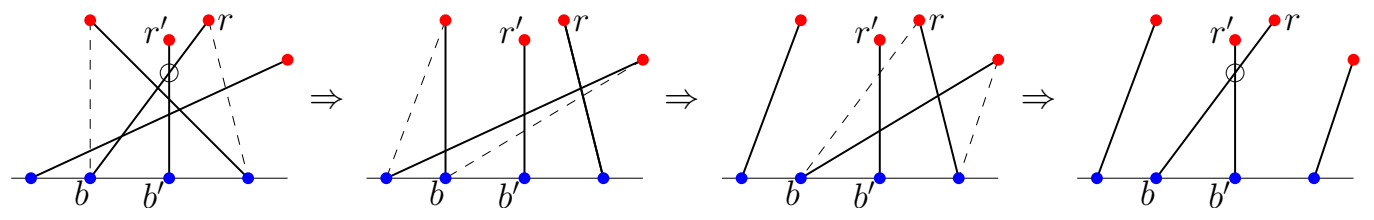

Figure 5 Reappearance of the crossing between $b r$ and $b^{\prime} r^{\prime}$.

For an illustration of the rest of the proof, follow Figure 4 . The sequence $p_{2}, \ldots, p_{m+1}$ starts with a red point and ends with a blue point. Thus, in this sequence there are two points of distinct colors, say $a$ and $b$, that are consecutive. Let $b$ be the first blue point after $p_{1}$. Let $a^{\prime}$ and $b^{\prime}$ be the two points that are matched to $a$ and $b$ respectively. By flipping $b b^{\prime}$ and $p_{1} p_{m+2}$ to $b^{\prime} p_{m+2}$ and $b p_{1}$, and then flipping $b p_{1}$ and $a a^{\prime}$ to $p_{1} a^{\prime}$ and $a b$ we obtain $M^{\prime}$ in which $a b$ is a boundary edge.

Let $M^{\prime \prime}$ be the bichromatic matching on $n-2$ points obtaining from $M^{\prime}$ by removing a boundary edge. By the induction hypothesis, it holds that

$$
f(M) \leqslant 2+f\left(M^{\prime \prime}\right) \leqslant 2+((n-2)-2)=n-2 .
$$

In the rest of this section we study the case where $P$ is semi-collinear, i.e., its blue points are on a straight line and its red points are in general position. Semi-collinear points have been studied in may problems related to plane matchings (see e.g., [5, 6, 10]). We prove that for every perfect bichromatic matching $M$ on $P$, it holds that $f(M)=O\left(n^{2}\right)$. Before we prove this upper bound, observe that similar to the general position setting, in the semi-collinear setting the total number of crossings might increase after a flip. Also, it is possible that a crossing, that has disappeared after a flip, reappears after some more flips (see the crossing between $b r$ and $b^{\prime} r^{\prime}$ in Figure 5). The $O\left(n^{2}\right)$ upper bound given in [7] for $f(\cdot)$ on uncolored points, which is obtained by connecting the two leftmost points of a crossing, does not apply to our semi-collinear bichromatic setting, because in this setting the two leftmost points might have the same color. These observations imply that there is no straightforward way of getting a good upper bound.

Let $\ell$ be the line that contains all the blue points of $P$. By a suitable rotation we assume that $\ell$ is horizontal. For every perfect bichromatic matching $M$ on $P$, the edges of $M$, that are above $\ell$, do not cross the ones that are below $\ell$. Thus, we can handle these two sets of edges independently of each other. Therefore, in the rest of this section we assume that the red points of $P$ lie above $\ell$. Recall that $P$ contains $n / 2$ blue points and $n / 2$ red points.

- Lemma 16. Let $M$ be a perfect bichromatic matching on $P$ in which the rightmost blue point $b$ is matched to the topmost red point $r$. If $M \backslash\{b r\}$ is plane, then $f(M) \leqslant \frac{n}{2}-1$, and this bound is tight.

Proof. See Figure 6(a) for an illustration of the statement of this lemma; notice that if we remove $b r$ from $M$, then we get a plane matching. Our proof is by induction on $n$. If $n=2$, then $M$ has one edge which is plane, and thus, $f(M)=0$. Assume that $n \geqslant 4$. If $b r$ does not intersect any other edge, then $M$ is plane and $f(M)=0$. Suppose that $b r$ intersects some edges of $M \backslash\{b r\}$, and let $R^{\prime}$ be the set of the red endpoints of those edges; see Figure 6(a). Let $r^{\prime}$ be the first red point in the counterclockwise order of the red points around $b$; observe that $r^{\prime}$ belongs to $R^{\prime}$. Let $b^{\prime}$ be the blue point that is matched to $r^{\prime}$. Flip $b r$ and $b^{\prime} r^{\prime}$ to $b r^{\prime}$ and $b^{\prime} r$ as in Figure $6(\mathrm{~b})$, and let $M^{\prime}$ be the resulting matching. The edge $b r^{\prime}$ does not cross any other edge of $M^{\prime}$, because of our choice of $r^{\prime}$, but the edge $b^{\prime} r$ may cross some edges of $M^{\prime}$. Let $M^{\prime \prime}$ be the subset of edges of $M^{\prime}$ that are to the left of $\overline{b^{\prime} r^{\prime}}$; see Figure $6(\mathrm{~b})$. Notice that $b r^{\prime} \notin M^{\prime \prime}$, and thus $M^{\prime \prime}$ is a matching on at most $n-2$ points. 


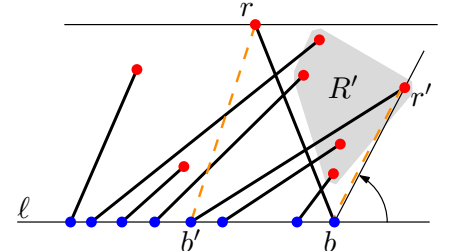

(a)

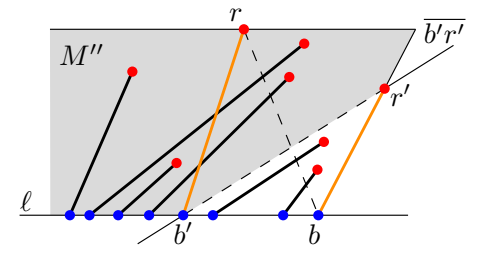

(b)

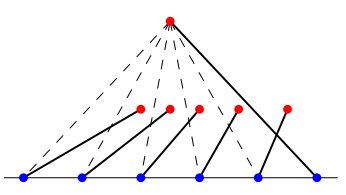

(c)

Figure 6 Illustration of the proof of Lemma 16.
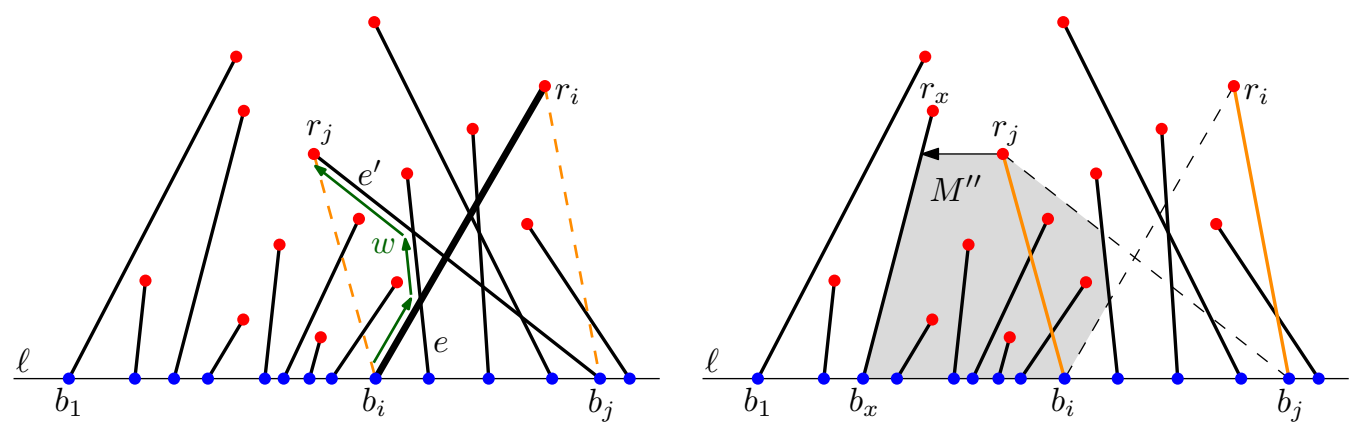

Figure 7 Illustration of the proof of Theorem 17.

Because of the planarity of $M \backslash\{b r\}$ and since $r$ is the topmost red point, we have that $M^{\prime} \backslash M^{\prime \prime}$ is plane. Moreover, $M^{\prime \prime}$ and $M^{\prime} \backslash M^{\prime \prime}$ are separated by $\overline{b^{\prime} r^{\prime}}$. Observe that $b^{\prime}$ is the rightmost blue point in $M^{\prime \prime}$ that is matched to the topmost red point $r$, moreover, $M^{\prime \prime} \backslash\left\{b^{\prime} r\right\}$ is plane. Therefore, we can repeat the above process on $M^{\prime \prime}$, which is a smaller instance of the initial problem. By the induction hypothesis, it holds that

$$
f(M)=1+f\left(M^{\prime \prime}\right) \leqslant 1+\left(\frac{n-2}{2}-1\right)=\frac{n}{2}-1 .
$$

To verify the tightness, Figure 6(c) shows a matching example for which we need exactly $n / 2-1$ flips to transform it to a plane matching. Each time there exists exactly one crossing, and after flipping that crossing, only one other crossing appears (except for the last flip).

- Theorem 17. For every perfect bichromatic matching $M$ on $P$ we have $f(M) \leqslant \frac{n^{2}}{8}+\frac{n}{4}$.

Proof. We present an iterative algorithm that uncrosses $M$ by $O\left(n^{2}\right)$ flips. Let $b_{1}, \ldots, b_{n / 2}$ be the blue points from left to right. By a suitable relabeling assume that $M=\left\{b_{1} r_{1}, \ldots\right.$, $\left.b_{n / 2} r_{n / 2}\right\}$. To simplify the description of the proof, we add, to $M$, a dummy edge $b_{0} r_{0}$ such that $b_{0}$ is a blue point on $\ell$ that is to the left of all the blue points, $r_{0}$ is a red point that is higher than all the red points, and all points of $P$ are to the right of $\overline{b_{0} r_{0}}$.

We describe one iteration of our algorithm. If $M$ is plane, then the algorithm terminates. Assume that $M$ is not plane. Let $i \in\{1, \ldots, n / 2\}$ be the smallest index such that $b_{i} r_{i}$ intersects some edges of $M$; see Figure 7-left. To simplify the rest of our description, we refer to the current iteration as iteration $i$. Notice that the blue endpoint of every non-free edge is strictly to the right of $b_{i-1}$. Let $r_{j}$ be the first red point that we meet in the following walk along the edges of $M$. Starting from $b_{i}$, we walk along $b_{i} r_{i}$ until we see the first edge $e$ that crosses $b_{i} r_{i}$. Then we turn left on $e$ and keep walking until we see a red point or another crossing edge. If we see a red point, then we call it $r_{j}$ and finish the walk. If we see a crossing edge $e^{\prime}$, then we turn left on $e^{\prime}$ and keep walking until we see a red point, namely $r_{j}$, or we see another crossing edge. In the latter case we repeat this process and stop as soon as we 
see the first red point, which we call it $r_{j}$. Let $b_{j}$ be the blue point that is matched to $r_{j}$. Let $w$ denote the convex polygonal path that we traversed from $b_{i}$ to $r_{j}$.

Flip $b_{i} r_{i}$ and $b_{j} r_{j}$ to $b_{i} r_{j}$ and $b_{j} r_{i}$, and let $M^{\prime}$ denote the resulting matching. See Figure 7-right. Shoot a horizontal ray, from $r_{j}$, to the left, and stop as soon as it hits an edge $b_{x} r_{x}$ in $M^{\prime}$. Let $M^{\prime \prime}$ be the subset of the edges of $M^{\prime}$ that are incident on $b_{x+1}, \ldots, b_{i}$, that is, $M^{\prime \prime}=\left\{b_{x+1} r_{x+1}, \ldots, b_{i-1} r_{i-1}, b_{i} r_{j}\right\}$. By the way that we picked $r_{j}$, the edges of $M^{\prime \prime}$ are in a convex region whose interior is disjoint from the edges of $M^{\prime} \backslash M^{\prime \prime}$; this convex region is bounded by $\ell, b_{x} r_{x}, w$, and the ray from $r_{j}$, as depicted in Figure 7-right. The matching $M^{\prime \prime}$ has $i-x$ edges. Observe that, in $M^{\prime \prime}$, we have that $b_{i}$ is the rightmost blue point that is matched to the topmost red point $r_{j}$, and $M^{\prime \prime} \backslash\left\{b_{i} r_{j}\right\}$ is plane. Thus, by Lemma 16 we can uncross $M^{\prime \prime}$ by at most $i-x-1$ flips. To this end, we have transformed $M$ to a matching in which the edges that are incident on $b_{1}, \ldots, b_{i}$ are free. The total number of flips performed in iteration $i$ is at most $1+(i-x-1)=i-x \leqslant i$.

In the next iteration, the smallest index $i^{\prime}$, for which $b_{i^{\prime}} r_{i^{\prime}}$ is not free, is larger than $i$. Thus, this smallest index moves at least one step to the right after each iteration. This means that the number of free edges, that are connected to the blue points of lower indices, increases. Therefore, after at most $n / 2$ iterations our algorithm terminates. The total number of flips is

$$
f(M) \leqslant \sum_{i=1}^{n / 2} i=\frac{n^{2}}{8}+\frac{n}{4}
$$

\section{Conclusions}

We investigated the number of flips that are necessary and sufficient to reach a non-crossing perfect matching on $n$ points in the plane. It is known that the minimum and the maximum lengths of a flip sequence are $O\left(n^{2}\right)$ and $O\left(n^{3}\right)$, respectively. We proved, with a new approach, that the minimum length of a flip sequence is $O(n \Delta)$ where $\Delta$ is the spread of the points set; this improves the bound for point sets with sublinear spread. A natural open problem is to improve any of these bounds. Another open problem is to improve our $O(n \log n)$ upper bound on the number of sufficient flips to reach a plane spanning tree on points in convex position, or to show that this bound is tight. One potential way to do this, is that in Theorem 14, we get a boundary edge $u v$ such that one of $u$ or $v$ has a constant degree.

It is worth mentioning that the number of flips, in a flip sequence, is highly dependent on the order in which we choose crossings to flip, and the type of a flip that we perform (among the two possible types). This dependency can be used to improve the bounds on the minimum number of flips. In Theorems 11, 14, 15, and 17 we used the order and proved some upper bounds, while in Theorem 4 we used the flip type. One may think of using the order and the flip type together to improve the current bounds. Notice that for bichromatic matchings, spanning trees, and Hamiltonian cycles only one type of flip is possible, and thus, only the order can be used for further improvements. Also, notice that none of the order and the flip type can be used to improve the bounds on the maximum number of flips, because, in this case, an adversary chooses the order and the type.

\section{References}

1 Oswin Aichholzer, Andrei Asinowski, and Tillmann Miltzow. Disjoint compatibility graph of non-crossing matchings of points in convex position. Electr. J. Comb., 22(1):P1.65, 2015.

2 Oswin Aichholzer, Sergey Bereg, Adrian Dumitrescu, Alfredo García Olaverri, Clemens Huemer, Ferran Hurtado, Mikio Kano, Alberto Márquez, David Rappaport, Shakhar 
Smorodinsky, Diane L. Souvaine, Jorge Urrutia, and David R. Wood. Compatible geometric matchings. Comput. Geom., 42(6-7):617-626, 2009.

3 Oswin Aichholzer, Wolfgang Mulzer, and Alexander Pilz. Flip distance between triangulations of a simple polygon is NP-complete. Discrete 63 Computational Geometry, 54(2):368$389,2015$.

4 Greg Aloupis, Luis Barba, Stefan Langerman, and Diane L. Souvaine. Bichromatic compatible matchings. Comput. Geom., 48(8):622-633, 2015.

5 Greg Aloupis, Jean Cardinal, Sébastien Collette, Erik D. Demaine, Martin L. Demaine, Muriel Dulieu, Ruy Fabila Monroy, Vi Hart, Ferran Hurtado, Stefan Langerman, Maria Saumell, Carlos Seara, and Perouz Taslakian. Non-crossing matchings of points with geometric objects. Computational Geometry: theory and Applications, 46(1):78-92, 2013.

6 Ahmad Biniaz, Anil Maheshwari, and Michiel Smid. Bottleneck bichromatic plane matching of points. In Proceedings of the 26th Canadian Conference on Computational Geometry ( $C C C G)$, pages 431-435, 2014.

7 Édouard Bonnet and Tillmann Miltzow. Flip distance to a non-crossing perfect matching. EuroCG, 2016.

8 Prosenjit Bose and Ferran Hurtado. Flips in planar graphs. Comput. Geom., 42(1):60-80, 2009.

9 Prosenjit Bose and Sander Verdonschot. A history of flips in combinatorial triangulations. In XIV Spanish Meeting on Computational Geometry (EGC), pages 29-44, 2011.

10 John Gunnar Carlsson, Benjamin Armbruster, Saladi Rahul, and Haritha Bellam. A bottleneck matching problem with edge-crossing constraints. International Journal of Computational Geometry and Applcations, 25(4):245-262, 2015.

11 Kenneth L. Clarkson. Nearest neighbor queries in metric spaces. Discrete \& Computational Geometry, 22(1):63-93, 1999.

12 Thomas H. Cormen, Charles E. Leiserson, Ronald L. Rivest, and Clifford Stein. Introduction to Algorithms, chapter 21: Data structures for Disjoint Sets. The MIT Press and McGrawHill Book Company, second edition, 2001.

13 Herbert Edelsbrunner, Pavel Valtr, and Emo Welzl. Cutting dense point sets in half. Discrete $\&$ Computational Geometry, 17(3):243-255, 1997.

14 Ferran Hurtado, Marc Noy, and Jorge Urrutia. Flipping edges in triangulations. Discrete E Computational Geometry, 22(3):333-346, 1999.

15 Mashhood Ishaque, Diane L. Souvaine, and Csaba D. Tóth. Disjoint compatible geometric matchings. Discrete \& Computational Geometry, 49(1):89-131, 2013.

16 Charles L Lawson. Transforming triangulations. Discrete Mathematics, 3(4):365-372, 1972.

17 Yoshiaki Oda and Mamoru Watanabe. The number of flips required to obtain non-crossing convex cycles. In Proceedings of the International Conference on Computational Geometry and Graph Theory (KyotoCGGT), pages 155-165, 2007.

18 Alfredo García Olaverri, Clemens Huemer, Ferran Hurtado, and Javier Tejel. Compatible spanning trees. Comput. Geom., 47(5):563-584, 2014.

19 Pavel Valtr. Planar point sets with bounded ratios of distances. PhD thesis, Fachbereich Mathematik, Freie Universität Berlin, 1994.

20 Pavel Valtr. Lines, line-point incidences and crossing families in dense sets. Combinatorica, 16(2):269-294, 1996.

21 Jan van Leeuwen and Anneke A. Schoone. Untangling a travelling salesman tour in the plane. In Proceedings of the 7th Conference Graphtheoretic Concepts in Computer Science $(W G)$, pages $87-98,1981$. 